\title{
Energy and Momentum Distribution of Surface Plasmon-induced Hot Carriers Isolated via Spatiotemporal Separation
}

\author{
Michael Hartelt, ${ }^{*}$ Pavel N. Terekhin, Tobias Eul, Anna-Katharina Mahro, \\ Benjamin Frisch, Eva Prinz, Baerbel Rethfeld, Benjamin Stadtmüller, and \\ Martin Aeschlimann \\ Department of Physics and Research Center OPTIMAS, TU Kaiserslautern, Germany \\ E-mail: hartelt@physik.uni-kl.de
}

August 2, 2021

\begin{abstract}
Understanding the differences between photoninduced and plasmon-induced hot electrons is essential for the construction of devices for plasmonic energy conversion. The mechanism of the plasmonic enhancement in photochemistry, photocatalysis, and light-harvesting and especially the role of hot carriers is still heavily discussed. The question remains, if plasmoninduced and photon-induced hot carriers are fundamentally different, or if plasmonic enhancement is only an effect of field concentration producing these carriers in greater numbers. For the bulk plasmon resonance, a fundamental difference is known, yet for the technologically important surface plasmons this is far from being settled. The direct imaging of surface plasmon-induced hot carriers could provide essential insight, but the separation of the influence of driving laser, field-enhancement, and fundamental plasmon decay has proven to be difficult. Here, we present an approach using a two-color femtosecond pump-probe scheme in time-resolved 2-photon-photoemission (tr2PPE), supported by a theoretical analysis of the light and plasmon energy flow. We separate the energy and momentum distribu-
\end{abstract}

tion of the plasmon-induced hot electrons from the one of photoexcited electrons by following the spatial evolution of photoemitted electrons with energy-resolved Photoemission Electron Microscopy (PEEM) and Momentum Microscopy during the propagation of a Surface Plasmon Polariton (SPP) pulse along a gold surface. With this scheme, we realize a direct experimental access to plasmon-induced hot electrons. We find a plasmonic enhancement towards high excitation energies and small in-plane momenta, which suggests a fundamentally different mechanism of hot electron generation, as previously unknown for surface plasmons.

\section{Keywords}

plasmon-induced hot carriers; surface plasmon polariton; PEEM; 2-photon photoemission; momentum microscopy

\section{Introduction}

The world's vital demand for clean energy poses a huge challenge for fundamental and application-oriented research to devise new and sustainable concepts to convert solar power into electric and chemical energy. One key perspective to enhance the efficiency of light-to- 
carrier conversion processes is plasmon technology. Despite the great potential of plasmoninduced hot carriers for photovoltaics and photochemistry, ${ }^{1-3}$ their precise role in the enhancement of the efficiency of these processes is still heavily discussed. ${ }^{1,4,5}$ Fundamental questions remain on how plasmon-induced hot carriers are generated, how they dissipate energy and momentum, and how the underlying mechanisms come into play in plasmonic energy conversion processes. Many theoretical studies have been conducted in the last years to address these questions. ${ }^{6-17}$ However, it is essentially unresolved if plasmonic enhancement is simply due to the field-enhancement of light at the surface of a metal, or if there is a more fundamental difference between plasmon-induced and photon-induced hot carriers.

For the bulk plasmon resonance at the plasma frequency $\omega_{\mathrm{p}},{ }^{18}$ a fundamental difference has long been known from theoretical studies ${ }^{19}$ and was more recently shown in linear ${ }^{20,21}$ and nonlinear ${ }^{22,23}$ photoemission experiments: Bulk plasmons selectively excite electrons from occupied states close to the Fermi energy $E_{\mathrm{F}}$ of the metal. This results in a peak in the photoemission spectrum at $E-E_{\mathrm{F}}=\hbar \omega_{\mathrm{p}}\left(\right.$ or $2 \hbar \omega_{\mathrm{p}}$ in the non-linear case). This energy-selective emission strongly contradicts the expectation for the electron and hole distributions in conventional photoexcitation, which are governed by the density of states (DOS) of the material. The effect was referred to as non-Einsteinian photoemission ${ }^{22-24}$ due to its fixed energetic position for all photon energies.

In energy harvesting applications, often energy thresholds have to be overcome for effective charge separation or driving a chemical reaction. Therefore, a preferential high-energetic electron excitation, as observed for bulk plasmons, could hold great potential. This would require that a similar effect also exists for surface plasmons, which can be excited at optical frequencies and concentrate the energy density at the metal surface, where electrons can be transferred across a functional interface or they can be excited directly via chemical interface damping. ${ }^{17,25} \mathrm{~A}$ theoretical model for the bulk case by Novko et al. suggests that a simi- lar plasmonic decay for surface plasmons might be possible. ${ }^{24}$ Although some experiments at nanostructure interfaces show an enhancement for high-energy electrons, ${ }^{26-28}$ a clear identification of the microscopic mechanisms was not yet possible due to the complex interplay of various experimental parameters (field enhancement, interface effects, laser field, etc.)

Conventionally, plasmonic excitations and their decay are investigated experimentally using optical spectroscopy techniques. ${ }^{29}$ In such measurements, different decay channels can be quantified by the systematic variation of parameters. ${ }^{30}$ The dynamics of the particularly intriguing hot electrons can be addressed, at least indirectly, via time-resolved spectroscopy techniques. ${ }^{31-33}$

A more direct access to the excited electron ("hot carrier") dynamics on the femtosecond timescale and in the single-particle limit can be gained by the time-resolved 2-photonphotoemission (tr-2PPE) technique. ${ }^{34-38}$ This method has been established since many years for the case of optical rather than plasmonic excitation, even in the context of hot carrier assisted photochemistry. ${ }^{39}$ On the other hand, plasmonic fields themselves have been successfully imaged with time-resolved Photoemission Electron Microscopy (tr-PEEM), ${ }^{40}$ both for localized $^{41-43}$ and propagating ${ }^{43-45}$ surface plasmons (LSP and SPP, respectively). In this context, the photoelectrons were used merely as an experimental observable for the plasmon field. Combining these two approaches of photoemission opens up ways towards imaging plasmoninduced hot carriers on the femtosecond and nanometer scale. However, the progress in this direction has been slow, as the question about the microscopic mechanism of surface plasmon damping arose two decades ago from early photoemission experiments on plasmonic samples. ${ }^{46}$ The separation of plasmon and electron dynamics was limited to describing the plasmon as a modified electromagnetic field in the vicinity of the surface, ${ }^{47}$ known as the plasmonic near-field.

In recent surface plasmon-induced photoemission experiments, ${ }^{26,28,48-51}$ it is still not easy to distinguish the plasmon effect from that of 
the driving laser, much less the field enhancement effect from the more fundamental plasmon decay. To acquire a photoemission signal dominated by a plasmonic field, a strong field-enhancement was used, either by using LSP resonances at nanostructures, ${ }^{26,28,49-51}$ or via the strong focusing of an SPP wave. ${ }^{48}$ Photoemission with contribution of an unfocused SPP wave is present in tr-PEEM, but its imaging requires the interference with a probe laser pulse. ${ }^{45}$ When the plasmon and the probe laser field coexist in space and time, a manifold of possible transition pathways with arbitrary, indistinguishable contributions of the two fields take part in photoexcitation into a state of given energy. ${ }^{52}$ This obscures the experimental access to the plasmon-induced hot electrons sought for.

In this paper, we employ two-color time- and energy-resolved PEEM experiments in real and momentum space to directly image plasmoninduced hot carriers. We demonstrate the separation of the photoelectrons generated in the process, in which first a plasmon decays by producing an excited (hot) electron, which is then photoemitted by a photon of double the energy. To achieve this, we use the spatiotemporal dynamics of a propagating SPP plane wave pulse. We perform a theoretical analysis of the flow of SPP energy density to separate the different contributing terms of SPP and laser analytically. Our method provides a direct imaging of plasmon-induced hot electrons, with sensitivity to the time, space, momentum, and energy domains.

\section{Results and Discussion}

\section{Spatiotemporal Separation Scheme}

Our experimental scheme to separate the photon- and plasmon-induced hot carriers is sketched in Figure 1. An ultrashort SPP pulse is excited at a coupling slit, engraved into a gold layer, oriented along the $y$-direction at $x=0 \mu \mathrm{m}$. The red pump pulse $(\tau \approx 23 \mathrm{fs}$, $\lambda_{\text {center }}=800 \mathrm{~nm} \Rightarrow h \nu=1.55 \mathrm{eV}$ ) is focused on the coupling structure under near-normal inci- dence, with linear polarization in $x$-direction, perpendicular to the slit. The structure constitutes a break of translation symmetry, providing additional momentum which allows the coupling of light into the SPP mode. In that way, an SPP pulse with a plane wavefront is launched, which then propagates along the $\mathrm{Au}$ vacuum interface in positive $x$-direction. ${ }^{53}$

The damping of the SPP is dominated by the internal decay in the metal, producing electronhole pairs, because of the nature of the SPP being a dark mode that cannot decay into the far-field without a break of symmetry at the surface. In this way, the SPP pulse acts as a propagating plasmonic source of hot electrons.

We probe the hot electron population by a time-delayed irradiation of the sample surface with a blue $\left(\lambda_{\text {center }}=400 \mathrm{~nm} \Rightarrow h \nu=3.1 \mathrm{eV}\right)$ laser pulse, generated by second harmonic generation from a split-off part of the fundamental output of the laser light source. Using a sub-monolayer of Cesium evaporated onto the sample, the work function of the $\mathrm{Au}$ surface was reduced to $\Phi \approx 3.4 \mathrm{eV}$. In this way, the hot electrons with an excitation energy of $E-E_{\mathrm{F}} \geq 0.3 \mathrm{eV}$ can be photoemitted by absorbing a photon from the probe pulse. A Photoemission Electron Microscope (PEEM) is used to image these electrons from the sample to a detector which is sensitive to their position and energy (see Methods). In this way, a 4-dimensional dataset of photoelectron yield $Y(x, y, E, \Delta t)$ was recorded by scanning the delay times between the pump and probe pulses and acquiring $\mathrm{x}-\mathrm{y}$ - and energy-resolved PEEM images for each time step.

The possible excitation channels which contribute to the measured photoelectron yield are shown in Figure 1E. In the dataset, the relative yield of the static 2PPE by the probe pulse ("static" channel) can be referenced and subtracted, and the dynamic signal with contribution of excitations with $1.55 \mathrm{eV}$, namely from the pump or SPP pulses, remains. For this signal, there are two participating channels, which differ in the temporal order of the two sequential excitation steps. Apart from the previously outlined red-blue channel, in which the excited electron from the red pump pulse or SPP is pho- 

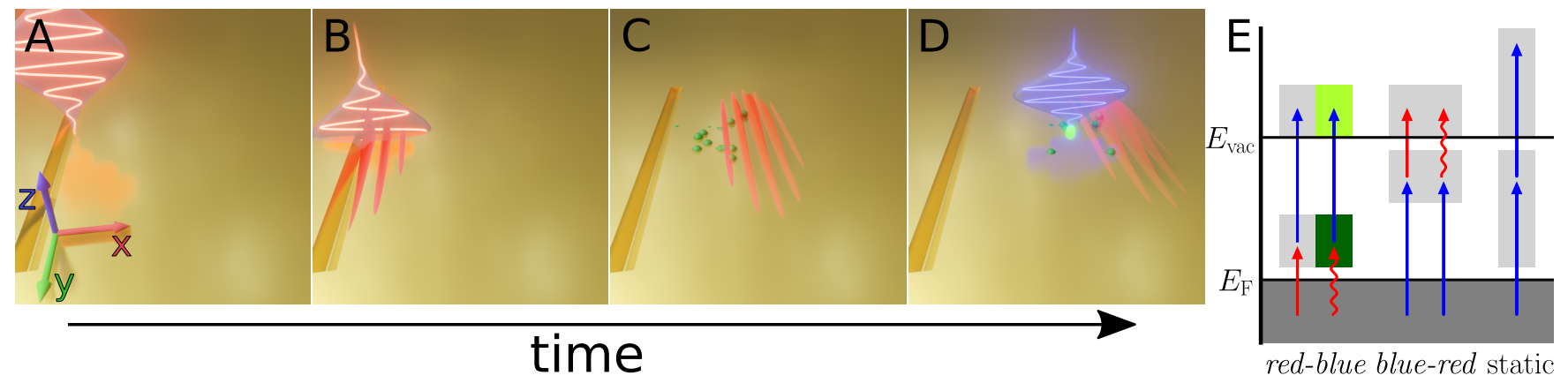

Figure 1: Scheme of spatiotemporal separation. A) The pump pulse (red) arrives at the coupling slit etched into the Au surface. B) An SPP pulse is excited and interacts with the remaining pump field during the pulse duration. C) The plasmon pulse propagates along the surface, exciting hot electrons (green balls) as it is damped through internal decay. D) The plasmon-excited hot electrons are photoemitted by a probe pulse (blue). A photoelectron (highlighted green ball) is produced, which is subsequently extracted and detected by the PEEM optics. The frames were taken from an animated video of the sequence, provided in the supplementary information. E) Energy diagram of possible 2PPE channels. Transitions are marked as arrows representing the driving force, straight red for the pump pulse, curly red for the SPP, and straight blue for the probe pulse. The relevant energy ranges of excited and photoemitted states are marked in green for the plasmon-induced hot carriers under investigation, or in light gray, respectively, for the other channels. The dark gray area represents occupied electronic states up to $E_{\mathrm{F}}$. The green highlighted channel is isolated by means of the spatiotemporal separation scheme.

toemitted by the blue probe, the reversed order is also possible, in which the blue probe laser excites an electron which is then photoemitted by the red pump laser or SPP (blue-red channel). The intermediate state of the blue-red channel is located at an energy $1.55 \mathrm{eV}$ higher than the one of the red-blue channel though. This state has an about ten times shorter inelastic lifetime, ${ }^{38}$ following essentially the well-known relation $T_{1} \propto\left(E-E_{\mathrm{F}}\right)^{-2}$ from Fermi liquid theory. Neglecting band structure effects, the 2PPE yield scales at least linearly ${ }^{54}$ with $T_{1}$, therefore, the red-blue channel dominates the signal.

The further isolation of the contribution of the plasmon-induced hot carriers from the ones excited directly by the pump laser is performed using the spatiotemporal signature of their source, namely the SPP pulse, which propagates along the surface of the sample on a femtosecond time scale. To characterize the spatiotemporal characteristics of this source as a reference for the following evaluation of the photoemission data, we analytically calculated the energy density flow of the time-dependent electromagnetic field in a model system that emulates the conditions in the experiment, but without a probe laser pulse (see Methods).

We have analytically derived the absorbed energy density rate by solving Maxwell's equations for the laser and SPP fields. This calculation describes the laser energy absorption and defines the spatiotemporal characteristics of the available energy for the excitation of hot electrons. We have shown in Ref. 55 that it contains different interference contributions (laser $\times$ laser, laser $\times S P P$, and $S P P \times S P P)$. Each of these terms contributes to the first step of the red-blue photoemission channel in Figure 1E, producing the hot electrons in the intermediate state of the process. The first term, laser $\times$ laser, has no plasmonic contribution and is responsible for purely photon-induced electrons. The second term, laser $\times S P P$, arises from the interference of the laser and SPP fields. We have shown in Ref. 52 with a one-color phase-resolved PEEM experiment that this term produces mixed contributions in the photoemission process where even entangled quantum pathways take part, 
in which both fields participate in an indistinguishable manner. This corresponds to a mixing between the two red-blue channels displayed in Figure 1E. Therefore, a hot electron produced by the laser $\times S P P$ term cannot be clearly classified as photon-induced or plasmoninduced. However, the third term, $S P P \times S P P$, describes the propagation and decay of the SPP pulse. This term is the origin of purely plasmon-induced hot carriers. A detailed description of the calculation and the resulting energy density rate in space and time is given in our previous work. ${ }^{55}$

The spatiotemporal dependence of the energy density rate at the interface between $\mathrm{Au}$ and vacuum is shown in Figure 2A, with the three separate contributing terms encoded in the red, green, and blue color channels, representing laser $\times$ laser, $S P P \times S P P$, and laser $\times S P P$, respectively. The pump pulse (red channel, laser $\times$ laser term) is visible as a nonpropagating signal around $t=0 \mathrm{fs}$. The SPP (green channel, $S P P \times S P P$ term) appears as a propagating trace in positive $x$-direction until well past the irradiation time. The interference term (blue channel, laser $\times S P P$ term) is present only where the two fields overlap in space and time, leading to a periodic variation from yellow to blue-shaded colors (inset in Figure 2A).

The dashed black line in Figure 2A represents the SPP group velocity. This velocity can be derived from the SPP dispersion relation as

$$
v_{\mathrm{SPP}}=\frac{d \omega}{d k_{\mathrm{SPP}}^{\prime}},
$$

where $k_{\mathrm{SPP}}^{\prime}$ is the real part of the SPP wave vector

$$
k_{\mathrm{SPP}}=\frac{\omega}{c_{0}} \cdot \sqrt{\frac{\epsilon_{\mathrm{m}} \epsilon_{\mathrm{d}}}{\epsilon_{\mathrm{m}}+\epsilon_{\mathrm{d}}}},
$$

where $\omega$ is the laser angular frequency, $c_{0}$ is the speed of light, and $\epsilon_{\mathrm{m}}$ and $\epsilon_{\mathrm{d}}$ are the dielectric functions of the metal and dielectric halfspaces, respectively.

\section{PEEM Results}

In the time-dependent energy density rate in Figure 2A, the laser and SPP contributions are clearly separated in time at larger distances from the excitation edge $(x \gtrsim 40 \mu \mathrm{m})$. During the pulse duration $\tau$, when the pump pulse impinges on the sample around $t=0 \mathrm{fs}$, the laser $\times$ laser term dominates. An electron which is photoemitted at this stage close to the excitation edge cannot be attributed unambiguously to either photonic or plasmonic excitation. In contrast, after the pulse has faded for $t>\tau$, the situation is different: The SPP pulse still propagates along the surface as a moving source, producing hot electrons along its way. An electron which is photoemitted and detected during this stage might still remain from a photonic excitation, but especially for higher energies where electron lifetimes are as short as only a few tens of fs, the SPP pulse is the predominant source of hot electrons.

The experimental PEEM data can be analyzed in terms of the same space-time characteristics to assign types of origin to the detected electrons. From the acquired $4 \mathrm{D}$ dataset of photoemission yield $Y(x, y, E, \Delta t)$, the contribution caused by the calculated dynamic source, $Y_{\text {source, }}$ is isolated by subtracting the static (delay-independent) 2PPE yield:

$$
\begin{aligned}
& Y_{\text {source }}(x, y, E, \Delta t) \\
& =Y(x, y, E, \Delta t) \\
& -Y_{\text {static }}(x, y, E) .
\end{aligned}
$$

The static yield $Y_{\text {static }}$ is derived from the relative signal before the two pulses overlap, by averaging the measured count values for the pulse delays in the range of $\Delta t<-200 \mathrm{fs}$. The only delay-independent 2PPE channel (static in Figure 1E) is the two-photon contribution from the probe pulse (blue-blue). It is the dominant contribution to $Y_{\text {static }}$. Additionally, a three-photon contribution from the pump pulse ("Red 3PPE") is present near the coupling slit, where the field of the pump pulse is strong, but its photoemission yield is small due to the higher-order photoemission process needed to overcome the work function. In the range of spatiotemporal separation $(x \gtrsim 40 \mu \mathrm{m})$, it is negligible. Real-space plots of $Y_{\text {static }}$ are available in the supplementary information.

The resulting dynamic photoemission yield 

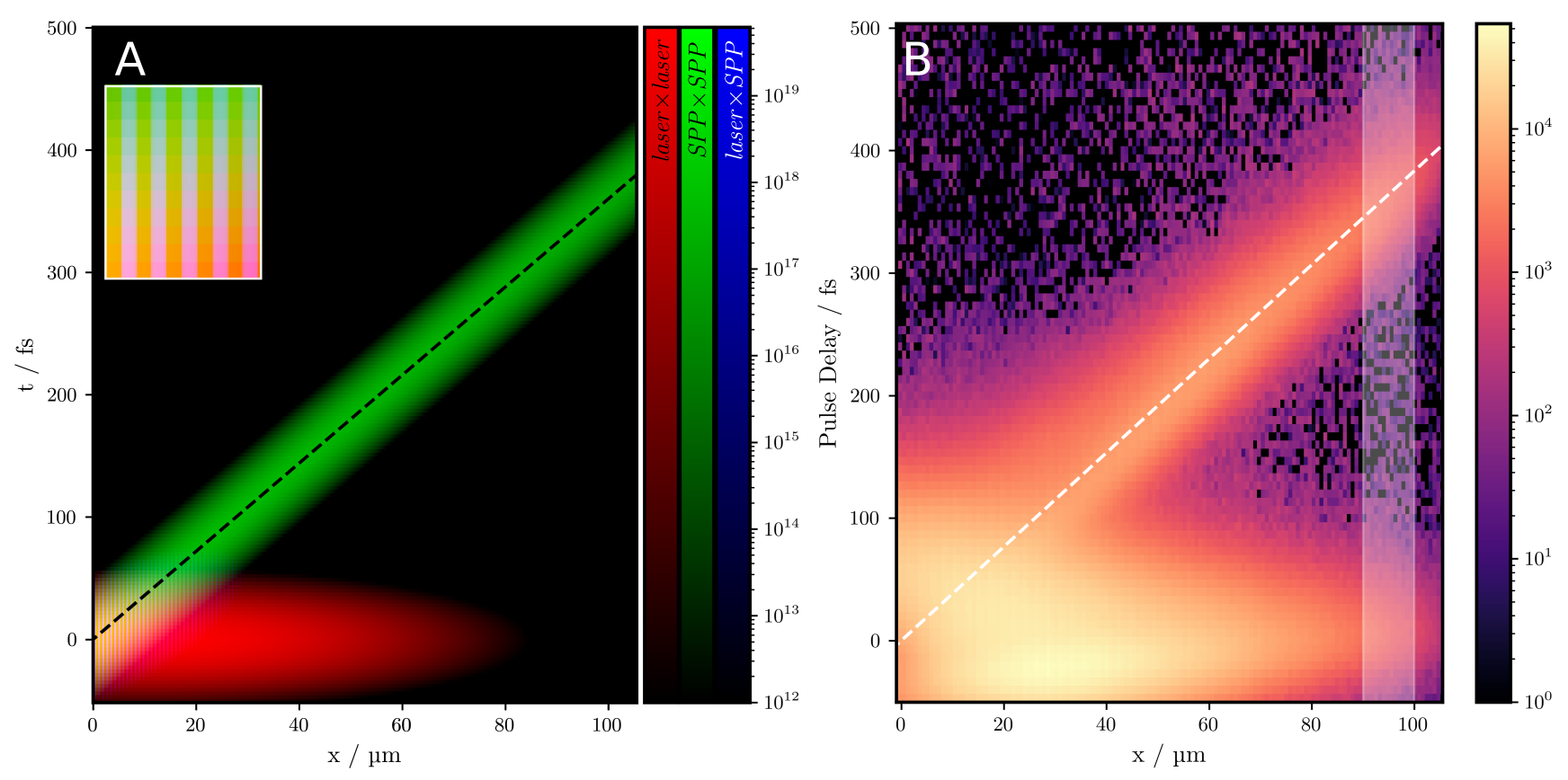

Figure 2: A) Time-dependent energy density rate of the electromagnetic field contributions at the surface, for a coupling structure at $x=0 \mu \mathrm{m}$ irradiated at normal incidence with a pump pulse centered at $t=0 \mathrm{fs}$. The three contributing terms, laser $\times$ laser, $S P P \times S P P$, and laser $\times S P P$ are encoded in the red, green, and blue color channels of the image, with each colorbar given in $\mathrm{J} \mathrm{s}^{-1} \mathrm{~m}^{-3}$. The additive color mixing leads to additional color shades where more than one field is present at the same time and position, which leads to yellow pixels for laser $\times$ laser and $S P P \times S P P$, and more blue-shaded colors for all three components. The velocity of an SPP $v_{\mathrm{SPP}}$ excited with $\lambda=800 \mathrm{~nm}$ is plotted as a dashed black line. Plots of the single components are available in the supplementary information. The inset is a zoom of $x=10 \mu \mathrm{m}$ to $15 \mu \mathrm{m}$ and $t=15 \mathrm{fs}$ to $45 \mathrm{fs}$, where all three components are present and the contribution of the interference term laser $\times S P P$ is visible as blue-shaded stripes. B) Measured tr-PEEM signal of the spatiotemporal signature of hot electrons, $Y_{\text {source, }}$, with the colorbar given in counts, at an excitation energy of $1.45 \mathrm{eV}$ to $1.55 \mathrm{eV}$, for an integration range of $y=55 \mu \mathrm{m}$ to $79 \mu \mathrm{m}$. The static background $Y_{\text {static }}$ was subtracted. The

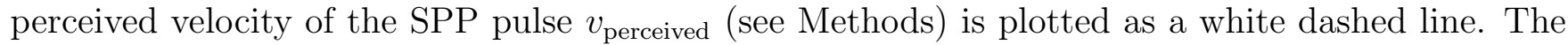
white shaded region represents the $x$ integration range for Figure 3. 
$Y_{\text {source }}(x, y, E, \Delta t)$ is shown in Figure $2 \mathrm{~B}$ as a 2D projection to the $x-\Delta t$ - plane. The integration range of $y=55 \mu \mathrm{m}$ to $79 \mu \mathrm{m}$ corresponds to the irradiated section of the coupling slit. The integration range in energy of $E-E_{\mathrm{F}}=1.45 \mathrm{eV}$ to $1.55 \mathrm{eV}$ corresponds to electrons excited from close to the Fermi edge. For these highest available excitation energies, the inelastic lifetime of hot electrons is shortest, in the order of tens of femtoseconds, ${ }^{38}$ and the trace is the least broadened in the time domain. Therefore, this range is best suited for comparison with Figure 2A to assign regions of predominant photonic and plasmonic excitation for the analysis of the respective electron energy distributions.

The obtained hot electron trace in Figure 2B can be clearly attributed to the spatiotemporal characteristics of the source: After an intense non-propagating feature that is caused by the pump pulse, a trace of electrons excited by the SPP is observed. For larger delays and distances from the coupling slit, the photon- and plasmon-induced hot electrons are clearly separated. The electrons observed in the propagating trace after the pump pulse has faded can be assigned to the photoemission channel in which the SPP contributes the initial excitation (green highlighted path in Figure 1E). This signal serves as an observable for the plasmoninduced hot electrons which are the main focus of this study.

Comparing the experimental PEEM trace to the temporal profile of the calculated energy density rate (Figure $2 \mathrm{~B}$ vs. 2A), the influence of the inelastic electron lifetime is visible in the slower temporal attenuation of the PEEM signal. An additional effect of broadening is added by the finite temporal length of the probe pulse, which universally provides a lower limit to the temporal width of a pump-probe signal, given by the cross-correlation between the two pulses.

For the study of plasmon-induced hot electrons, and in contrast to previously reported two-color PEEM experiments with SPP, ${ }^{56}$ the key in our isolation scheme is the combination of information of all available dimensions, in our case time, space, and energy. In the full 4-dimensional dataset, different aspects of spectral and lifetime information can be extracted

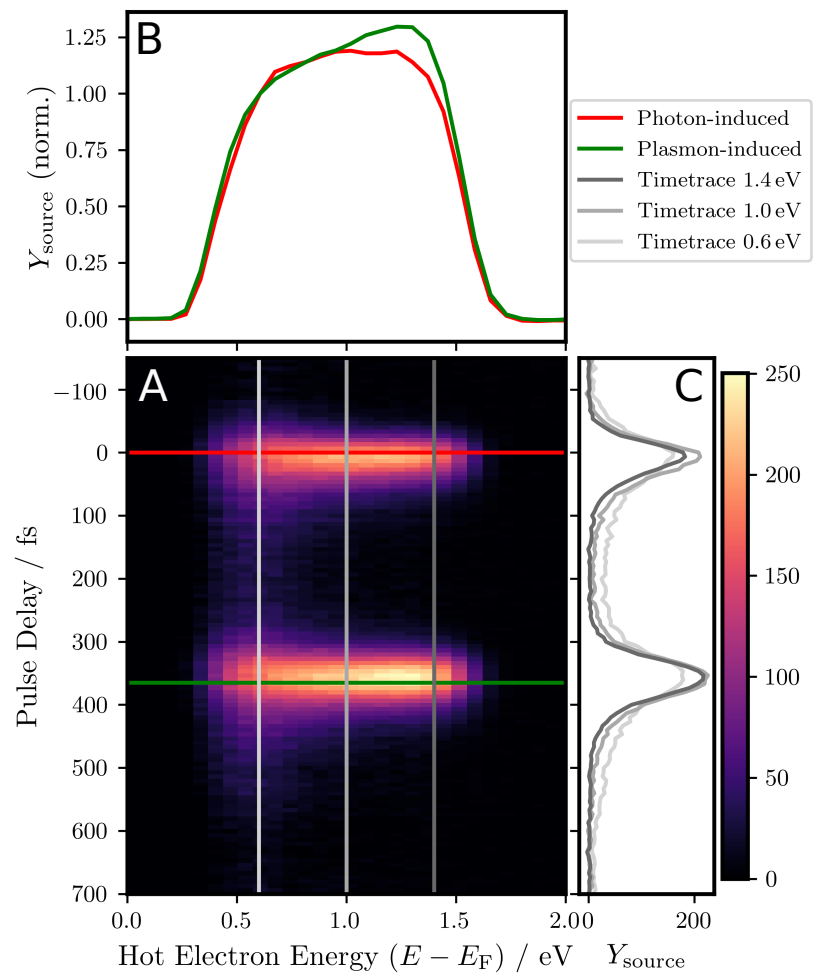

Figure 3: A) Projection of the 4D-dataset of photoemission yield for $x=90 \mu \mathrm{m}$ to $100 \mu \mathrm{m}$, $y=55 \mu \mathrm{m}$ to $79 \mu \mathrm{m}$ to the time-energy plane. B) Spectra of photon-induced (red line) and plasmon-induced (green line) hot electrons, extracted along the respective lines in A) at $\Delta t_{0}=$ 0 fs (red) and $\Delta t_{\mathrm{SPP}}=365 \mathrm{fs}$ (green), and normalized to the onset of the low-energy cutoff at $E-E_{\mathrm{F}}=0.6 \mathrm{eV}$. C) Time trace of electrons emitted from excitation energies of $E-E_{\mathrm{F}}=$ $1.4 \mathrm{eV}, 1.0 \mathrm{eV}$, and $0.6 \mathrm{eV}$, extracted as cuts along the respective gray lines in A). The colorbar and $Y_{\text {source }}$ axis values are given in counts. 
by evaluating different regions of the hypercube. This can be seen in the projection of certain slices of the dataset:

In the range of $x=95 \mu \mathrm{m}$, where the photonand plasmon contributions are most clearly separated in space and time (white shaded range in Figure 2B), a projection of the experimental 4D dataset to the $E$ - $\Delta t$-plane was plotted in Figure $3 \mathrm{~A}$. Line cuts were extracted using a temporal integration range of $\pm 25 \mathrm{fs}$ and an energy integration range of $\pm 0.05 \mathrm{eV}$. These cuts represent electron spectra for specific times (green, red, Figure 3B) and time traces for constant energies (gray, Figure 3C), respectively.

In the time traces (in Figure 3C), the typical lifetime behavior of hot electrons, governed by the Fermi liquid theory, ${ }^{38}$ is visible: The inelastic lifetimes $T_{1}$ increase with smaller excitation energy, which leads to more electrons remaining for later times after excitation (lighter gray compared to darker gray graphs).

In the energy domain, a photon-induced and a plasmon-induced hot electron spectrum is plotted for delay times of $\Delta t_{0}=0 \mathrm{fs}$ (red) and $\Delta t_{\mathrm{SPP}}=365 \mathrm{fs}$ (green), representing the stages at which the respective photonic and plasmonic contributions to the source field are most dominant. The spectra in Figure 3B are normalized to their low-energy cutoff (for absolute values see raw spectra in the supplementary information). A comparison shows that the higher excitation energies appear stronger in the plasmon-induced spectrum (see also difference spectrum in the supplementary information). This apparent preference for higherenergetic excitation is intriguing: Given that the photonic signal follows the familiar excitation mechanism with the energy distribution governed by the DOS of the material, it suggests that for the plasmonic signal a different excitation mechanism is involved. The highenergy feature emerging among the still present, continuous spectrum of photon-induced electrons hints that both the familiar single-particle excitation and a different plasmonic excitation might contribute. This would reflect the hybrid nature of the surface plasmon polariton as an electromagnetic wave (polariton aspect) bound to a collective charge motion (plasmon aspect). Following this reasoning, the polariton aspect would cause an Einsteinian photoexcitation known from light, while the plasmon aspect selectively excites electrons close to $E_{\mathrm{F}}$, similar to the effect known from bulk plasmons. ${ }^{19-24}$

An influence of the weaker blue-red channel should manifest in the time-resolved signal predominantly at time steps where the blue pulse precedes the red, for $\Delta t \lesssim \Delta t_{0}$ or $\Delta t \lesssim \Delta t_{\mathrm{SPP}}$, owing to the order of the involved excitation processes. Especially in the lower energy channels, secondary electrons would be expected due to the shorter lifetime of the intermediate state as discussed above. Such an influence is not observed in the electron traces, which leads us to conclude that the channel is not of significant strength, confirming that the observed hot electron distributions are predominantly excited by the pump and SPP pulses.

\section{Momentum Microscopy}

To gain further insight into the intriguing feature of high-energy preference of SPP-induced hot electrons, the momentum microscopy mode of the PEEM ${ }^{57-60}$ was used to characterize the distribution of plasmon-induced hot electrons in energy and momentum space ( $k$-space).

To use the concept of spatiotemporal separation in this mode, the probe laser focus was positioned in the center of the field-of-view, $\sim 100 \mu \mathrm{m}$ to the right of the coupling slit, and an iris aperture in the image plane of the electronoptical column was closed to limit the detection area to a diameter of $\sim 30 \mu \mathrm{m}$ in real space. In this way, the SPP pulse propagates through the selected area after the pump pulse is no longer present on the sample, similar to the temporal evolution in the white-shaded area of Figure 2 B.

The momentum space distribution of the emitted electrons was detected by imaging the back-focal plane of the objective lens of the PEEM to the detector, thus acquiring a $k$-space photoemission dataset of the shape $Y\left(k_{x}, k_{y}, E, \Delta t\right)$. Similarly to the subtraction of the static yield in equation (3), the signal contribution caused by the SPP source field (green highlighted path in Figure 1E) was extracted 

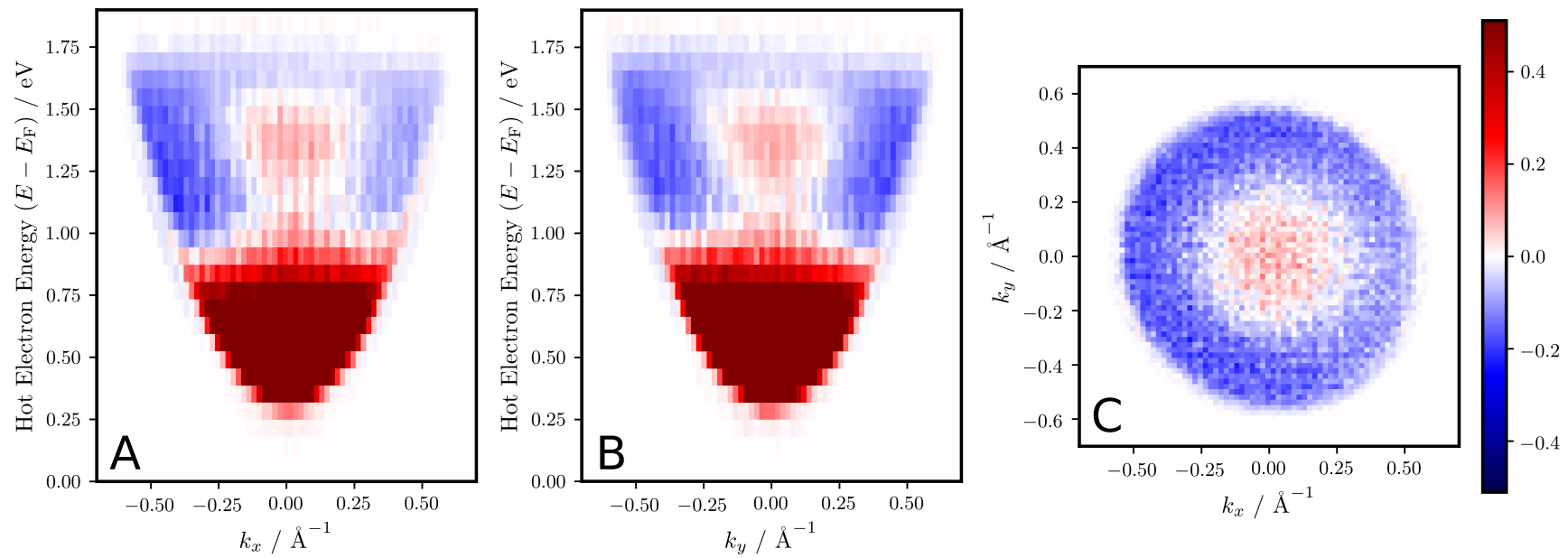

Figure 4: Momentum distribution of plasmon-induced hot electrons relative to photon-induced hot electrons. Cuts of the normalized difference values $\Delta Y\left(k_{x}, k_{y}, E\right)$ are shown along A) the $E$ - $k_{x^{-}}$ direction for $\left.k_{y}=0 \AA^{-1}, \mathrm{~B}\right)$ the $E$ - $k_{y}$-direction for $k_{x}=0 \AA^{-1}$, and C) the $k_{x}-k_{y}$-direction for $E-E_{\mathrm{F}}=1.4 \mathrm{eV}$ to $1.5 \mathrm{eV}$.

using the delay time when the SPP pulse is centered on the selected area, $\Delta t_{\mathrm{SPP}}=360 \mathrm{fs}$, and subtracting the static yield before the temporal overlap at $\Delta t_{\text {static }}=-334 \mathrm{fs}$ :

$$
\begin{aligned}
& Y_{\mathrm{SPP}}\left(k_{x}, k_{y}, E\right) \\
& =Y\left(k_{x}, k_{y}, E, \Delta t_{\mathrm{SPP}}\right) \\
& -Y\left(k_{x}, k_{y}, E, \Delta t_{\text {static }}\right) .
\end{aligned}
$$

A reference momentum distribution for photon-induced electrons $Y_{\text {photon }}$ (grey in redblue channel in Figure 1E) was measured with the red pump laser also centered to the fieldof-view instead of focused on the excitation slit, using identical sample position and PEEM settings. The pump-induced signal contribution was extracted at time zero $\Delta t_{0}=0 \mathrm{fs}$, subtracting the static probe yield in the same way:

$$
\begin{aligned}
& Y_{\text {photon }}\left(k_{x}, k_{y}, E\right) \\
& =Y_{\text {ref }}\left(k_{x}, k_{y}, E, \Delta t_{0}\right) \\
& -Y_{\text {ref }}\left(k_{x}, k_{y}, E, \Delta t_{\text {static }}\right),
\end{aligned}
$$

where $Y_{\text {ref }}$ is the photoelectron yield of the reference measurement.

Figure 4 shows the normalized difference $\Delta Y\left(k_{x}, k_{y}, E\right)$ between plasmon-induced and photon-induced hot electrons in momentum space. The values were normalized by

$$
\Delta Y\left(k_{x}, k_{y}, E\right)=\frac{Y_{\mathrm{SPP}}}{\left\langle Y_{\mathrm{SPP}}\right\rangle}-\frac{Y_{\text {photon }}}{\left\langle Y_{\text {photon }}\right\rangle},
$$

where $\left\langle Y_{\mathrm{SPP}}\right\rangle$ and $\left\langle Y_{\text {photon }}\right\rangle$ are the respective mean values of $Y_{\text {SPP }}$ and $Y_{\text {photon }}$ over the full energy and momentum range, to account for the arbitrary difference in absolute signal strength. Plots of the same $k$-space cuts of the individual yields $Y_{\text {SPP }}$ and $Y_{\text {photon }}$ are provided in the supplementary information.

The high-energy feature described in the previous section is present in the $k$-space data in Figure 4 as a contiguous red region in the energy range of $1.2 \mathrm{eV}$ to $1.55 \mathrm{eV}$ in the center of the momentum range, close to the $\bar{\Gamma}$-point of the surface. In this region, the value of $\Delta Y\left(k_{x}, k_{y}, E\right)$ is positive, corresponding to an enhanced generation of hot electrons by plasmons with respect to photons.

From the usual perspective of surface science, where one typically explains features in $k$-space in terms of the electronic band structure, this feature is surprising: Due to the polycrystalline nature of the sample and the consequent randomness of crystallite orientations contained in the detection area, band structure effects should average out and one expects a homogeneous momentum distribution of electrons. 
Interestingly, no significant anisotropy or asymmetry of the plasmon-induced electrons along the propagation direction of the SPP $\left(k_{x^{-}}\right.$ direction) is observed. The enhancement is concentrated at small values of the in-plane momenta $k_{\|}=\left(k_{x}^{2}+k_{y}^{2}\right)^{1 / 2}$, but otherwise symmetric in $k$-space. This implies that the wave vector orientation and related momentum of the SPP wave seems to have no relevant influence.

The obtained momentum distribution could be an important clue for a different mechanism taking part in electron excitation by surface plasmons. The transfer of energy from the collective to single-particle excitation seems to efficiently couple to electrons with low momentum and close to the Fermi surface. Possible origins of this phenomenon are to be looked for in the nature of the SPP: Apart from the difference in the orientation of oscillating electric and magnetic fields, the movement of electrons as part of the collective excitation (plasmon aspect) in contrast to the single-particle case could be crucial. But ultimately, at this point a conclusive explanation of the plasmon decay mechanism, which links the collective motion to the single particle momentum, is missing.

In Figure $4 \mathrm{~A}$ and $\mathrm{B}$, which show the normalized difference in the energy vs. momentum distribution in both directions $\left(E-k_{x}\right.$-plot and $E-k_{y}$-plot), a strong enhancement for smaller energies of $E-E_{\mathrm{F}}<0.8 \mathrm{eV}$ is also apparent, which seems to contradict the result of the real space experiment. It turns out though, that this is an artifact of the measurement parameters: In contrast to the pump pulse, which impinges on the sample under near-normal incidence and is present only for the laser pulse duration, the SPP pulse propagates with finite velocity through the detection area selected by the iris aperture. This results in an apparent broadening of the SPP pulse duration, increasing the detection probability for secondary electrons, which are produced by inelastic decay after the initial excitation. We show in the supplementary information that this effect can be mitigated using a smaller aperture size and extracting the data from time windows of the full time-resolved measurement, selected to compensate for the apparent broadening. Un- fortunately, the use of a very small aperture causes a different artifact in the electron-optics, therefore, we chose to show the present data in the main manuscript.

\section{Conclusion}

To summarize, we have shown how direct experimental access to plasmon-induced hot electrons, separated from the influence of driving laser and field enhancement effects, was realized in a photoemission experiment. This provides a novel opportunity for the exploration of plasmon-enhanced energy conversion and chemistry from the fundamental perspective of solidstate physics.

The results show a preference for higherenergetic excitations with small in-plane momentum induced by surface plasmons in contrast to photons, independent of the crystal orientation of the metal surface. This suggests that the mechanism of plasmon-enhanced energy harvesting is more fundamentally linked to the nature of the surface plasmon and its decay into hot carriers, rather than just a simple field-enhancement at the metallic surface. At this point it is unclear if the observed effect is of related physical origin as the similar-looking effect known for bulk plasmons. ${ }^{19-24}$ The aspect of a collective oscillation of electrons in both bulk and surface plasmons leads to the hypothesis that a similar mechanism may be at play. On the other hand, it must be noted that many aspects are fundamentally different: While the bulk plasmon is in its nature a resonance effect of the bulk electronic system, the SPP case constitutes a decay of surface plasmons at the metal-dielectric interface and far from resonance. In both cases, the preferential excitation of electrons at $E_{\mathrm{F}}$ is evident, but as the SPP follows the frequency of the pump light rather than being pinned to a resonance frequency, the non-Einsteinian characteristic of subsequent pinning of electron energy off from $\hbar \omega$ of the driving laser is not to be expected for the SPP case.

Irrespective of fundamental origin, a preferential generation of high-energy electrons holds 
great potential for chemical and energy harvesting purposes. Unlike the typical continuous distribution of energy to electrons and holes, here we have a case in which a concentration of energy to "hot" electrons with only low-energetic ("cold") holes is evident, which could be of key advantage. For technical applications, a promising decay process is the chemical interface damping ${ }^{17,25,61}$ at a metal interface, where carriers are directly excited in the adjacent material. It is yet to be determined whether the observed effect could play a role in this context, but an enhancement of plasmonic excitation for electrons at $E_{\mathrm{F}}$ was also reported for plasmonic nanoparticle interfaces. ${ }^{26-28} \mathrm{On}$ the other hand, the electronic structure at the chemical interface in such systems additionally provides emerging dephasing pathways, which are of importance for plasmon decay, ${ }^{17,62}$ as well as the dynamics of hot electrons at the interface. ${ }^{63}$ In fact, emergent plasmonic excitation features were explained in terms of interface effects in most previous work. The concept of spatiotemporal separation will bring further insight to the multitude of influential effects for plasmon damping and plasmonic excitation at such interfaces by making the plasmon-induced carriers directly experimentally accessible.

\section{Methods}

\section{Time-dependent Energy Density Calculations}

A detailed description of the calculation method is given in our previous work. ${ }^{55}$ In our simulations, we consider a Gaussian pulse profile for the incoming laser field, including all components of electric and magnetic fields. The system consists of the gold sample filling the half-space $z \leq 0$ and a perfect vacuum filling the other half-space $z>0$. As a spatially defined coupling structure providing SPP, we use a step edge perpendicular to the interface of height $50 \mathrm{~nm}$ at $x=0 \mu \mathrm{m}$ irradiated at normal incidence.

The laser parameters applied in the simulations are a laser wavelength centered at $\lambda=800 \mathrm{~nm}$, a pulse duration of $\tau=23 \mathrm{fs}$, a Gaussian width of $25 \mu \mathrm{m}$ centered at $x=10 \mu \mathrm{m}$ away from the position of the step edge and a pump pulse energy of $1 \mathrm{~nJ}$. The pulse arrives at the sample surface centered with its maximal amplitude at $t=0 \mathrm{fs}$. The coupling parameter at the step edge, $\beta$, is set to 0.2 . It describes the ratio of moduli of the SPP magnetic field amplitude and the incident laser magnetic field amplitude at the origin of the step edge $(x=0)$. The obtained energy density rate was averaged over one full period of the oscillating fields to account for the lack of phase resolution of the experiment.

We represent the dielectric half-space by a perfect vacuum with $\epsilon_{\mathrm{d}} \equiv 1$. The metallic halfspace is modeled with the dielectric function reported by Olmon et al. ${ }^{64}$ for evaporated gold.

\section{Sample}

The sample was produced in the Nano Structuring Center (NSC) at TU Kaiserslautern. A layer of gold with a thickness of $250 \mathrm{~nm}$ was sputter-deposited onto a substrate cut from a native-oxidized Silicon wafer. The straight coupling slit with a width of $\sim 100 \mathrm{~nm}$, a depth of $\sim 230 \mathrm{~nm}$, and a length of $80 \mu \mathrm{m}$ was etched by focussed ion beam (FIB) milling. A submonolayer of Cesium was evaporated onto the sample surface in situ to reduce the work function for the PEEM measurement.

\section{Two-color Pump-Probe Setup}

A femtosecond Ti:Sapphire laser (Spectra Physics Tsunami) produces pulses at a central wavelength of $\lambda=800 \mathrm{~nm}$ with a pulse duration down to $\tau=23 \mathrm{fs}$. In a home-built two-color time-resolved optical setup, after using a prism compressor for dispersion compensation, the pulses are split up in two arms of an interferometer. In one arm the ("probe") pulse is frequency-doubled with a beta barium borate (BBO) crystal and compressed again with another prism pair, then it is routed over a linear delay stage to adjust the delay between the pump and probe. The "pump" arm passes a fixed optical route of equal effective length. 
The two arms are subsequently recombined and irradiated onto the sample in the PEEM setup.

\section{Photoemission Electron Microscopy}

We use a customized commercial PEEM setup (IS-PEEM, Focus GmbH), which is designed for laser irradiation under near-normal incidence. ${ }^{44}$ The laser beam is routed over a small mirror, introduced into the electron column of the microscope near the optical axis, which leads to an angle of incidence $\mathrm{AOI} \approx 4^{\circ}$.

The beam diameters on the sample are approximately $50 \mu \mathrm{m}$, the pump beam centered at the excitation slit and the probe beam offset to $x \approx 70 \mu \mathrm{m}$ for the real space experiments and $x \approx 100 \mu \mathrm{m}$ for the momentum microscopy experiments.

The photoelectrons are detected with a delayline detector (DLD), which records their positions as well as their time-of-flight, with reference to a trigger signal from the laser. Thus, the kinetic energy of each photoelectron is measured through proper energy-calibration of the detector's time channels, and a time- and energy-resolved PEEM experiment ${ }^{65}$ is performed. The PEEM can be operated as a momentum microscope by imaging the back-focal plane of the objective lens, in this way detecting the angular distribution of photoelectrons.

In the real-space experiment, the SPP trace is observed through the probe pulse, which hits the sample under near-normal, but not perfectly normal incidence of AOI $\approx 4^{\circ}$. Therefore, the in-plane component of the wave vector of the probe pulse $k_{\|}=-\omega / c_{0} \cdot \sin (\mathrm{AOI})$ contributes to the perceived propagation. For the dashed white line in Figure 2B, this was taken into account by plotting the perceived SPP velocity (see derivation in SI)

$$
v_{\text {perceived }}=v_{\mathrm{SPP}} \cdot \frac{1}{1+\frac{v_{\mathrm{SPP}}}{c_{0}} \sin (\mathrm{AOI})},
$$

which is slightly slower than the real SPP velocity $v_{\text {SPP }}$.

\section{Data Evaluation}

For all tr-PEEM datasets, several runs of exposures for the same list of pump-probe delays were acquired and and summed up during postprocessing to improve the data quality.

The energy axis offset of all datasets was calibrated to the excited electron energy by fitting a Fermi distribution to the high-energy cutoff of the photoelectron spectrum acquired with the blue probe laser. The fitted Fermi energy was set to $E-E_{\mathrm{F}}=3.1 \mathrm{eV}$ according to the photon energy of the laser.

The real space data in Figures $2 \mathrm{~B}$ and 3 were binned in groups of $(2,16)$ pixels in $(x, y)$ and a constant background across energy channels was subtracted for each binned pixel prior to the subtraction of the static signal in equation (3) to increase the signal-to-noise ratio.

The $k$-scale in the momentum space measurements (Figure 4) was calibrated by fitting a parabolic free-electron dispersion to the lowenergy cutoff of the data in $E$ - $k_{x^{-}}$and $E-k_{y^{-}}$cuts of the center of the data.

For the calculation in equation (6), the measured reference data was slightly shifted by $(-0.5,2.0,0.15)$ pixels in $\left(k_{x}, k_{y}, E\right)$ to correct for small differences in alignment. To reduce noise, a Gaussian filter with a kernel (sigma) of $\left(0.005 \AA^{-1}, 0.005 \AA^{-1}, 0.2\right.$ energy channels) in $\left(k_{x}, k_{y}, E\right)$ was applied, then both datasets were binned in groups of $(8,8)$ pixels in $\left(k_{x}\right.$, $k_{y}$ ). To select only the region of statistically relevant data, voxels with less than 80 counts in $Y_{\mathrm{SPP}}$ or $Y_{\text {photon }}$ were ignored.

\section{Data Availability}

All calculated and experimental raw data from which the material in this work was evaluated is available on doi:10.5281/zenodo. 5102762

Acknowledgement This work is funded by the Deutsche Forschungsgemeinschaft (DFG, German Research Foundation) - TRR 173 268565370 (Projects A02 and B11) and project RE1141/14-2. We thank the Nano Structuring Center (NSC) at TU Kaiserslautern for sample preparation. Some simulations were executed on the high-performance cluster "El- 
wetritsch" through the projects TopNano and Mulan at TU Kaiserslautern, which is a part of the "Alliance of High Performance Computing Rheinland-Pfalz". We kindly acknowledge the support of Regionales Hochschulrechenzentrum Kaiserslautern. E.P. acknowledges support from the Max Planck Graduate Center with the Johannes Gutenberg University Mainz and TU Kaiserslautern through a PhD fellowship. M.H. would like to thank Benito Arnoldi and Andrew Price for help with 3D design and animation in Blender.

\section{Supporting Information Avail- able}

Real space PEEM images, plots of the individual source term components, derivation of the observed SPP velocity, raw electron spectra and difference spectrum, individual momentum microscopy data of the photon and SPP contributions, additional momentum microscopy experimental results with mitigation of the secondary electron artifact, movies of an animated spatiotemporal separation scheme and the time series of real space PEEM data.

\section{References}

1. Brongersma, M. L.; Halas, N. J.; Nordlander, P. Plasmon-induced hot carrier science and technology. Nature Nanotechnology 2015, 10, 25-34.

2. Linic, S.; Christopher, P.; Ingram, D. B. Plasmonic-metal nanostructures for efficient conversion of solar to chemical energy. Nature Materials 2011, 10, 911-921.

3. Atwater, H. A.; Polman, A. Plasmonics for improved photovoltaic devices. Nature $M a-$ terials 2010, 9, 205-213.

4. Aizpurua, J.; Baletto, F.; Baumberg, J.; Christopher, P.; de Nijs, B.; Deshpande, P.; Fernandez, Y. D.; Fabris, L.; Freakley, S.; Gawinkowski, S.; Govorov, A.; Halas, N.; Hernandez, R.; Jankiewicz, B.; Khurgin, J.;
Kuisma, M.; Kumar, P. V.; Lischner, J.; Liu, J.; Marini, A. et al. Theory of hot electrons: general discussion. Faraday Discussions 2019, 214, 245-281.

5. Baumberg, J. J. Hot electron science in plasmonics and catalysis: what we argue about. Faraday Discussions 2019, 214, 501-511.

6. Manjavacas, A.; Liu, J. G.; Kulkarni, V.; Nordlander, P. Plasmon-Induced Hot Carriers in Metallic Nanoparticles. ACS Nano 2014, 8, 7630-7638.

7. Sundararaman, R.; Narang, P.; Jermyn, A. S.; Goddard III, W. A.; Atwater, H. A. Theoretical predictions for hot-carrier generation from surface plasmon decay. Nature Communications 2014, 5, 5788 .

8. Zhang, H.; Govorov, A. O. Optical Generation of Hot Plasmonic Carriers in Metal Nanocrystals: The Effects of Shape and Field Enhancement. The Journal of Physical Chemistry C 2014, 118, 7606-7614.

9. Bernardi, M.; Mustafa, J.; Neaton, J. B.; Louie, S. G. Theory and computation of hot carriers generated by surface plasmon polaritons in noble metals. Nature communications 2015, 6 .

10. Brown, A. M.; Sundararaman, R.; Narang, P.; William A. Goddard, I.; Atwater, H. A. Nonradiative Plasmon Decay and Hot Carrier Dynamics: Effects of Phonons, Surfaces, and Geometry. ACS Nano 2016, 10, 957-966.

11. Saavedra, J. R. M.; Asenjo-Garcia, A.; García de Abajo, F. J. Hot-Electron Dynamics and Thermalization in Small Metallic Nanoparticles. ACS Photonics 2016, 3, 1637-1646.

12. Sykes, M. E.; Stewart, J. W.; Akselrod, G. M.; Kong, X.-T.; Wang, Z.; Gosztola, D. J.; Martinson, A. B. F.; Rosenmann, D.; Mikkelsen, M. H.; Govorov, A. O.; Wiederrecht, G. P. Enhanced 
generation and anisotropic Coulomb scattering of hot electrons in an ultrabroadband plasmonic nanopatch metasurface. Nature Communications 2017, 8.

13. Khurgin, J. B. Hot carriers generated by plasmons: where are they generated and where do they go from there? Faraday Discussions 2019, 214, 35-58.

14. Aguirregabiria, G.; Marinica, D.-C.; Ludwig, M.; Brida, D.; Leitenstorfer, A.; Aizpurua, J.; Borisov, A. G. Dynamics of electron-emission currents in plasmonic gaps induced by strong fields. Faraday Discussions 2019, 214, 147-157.

15. Do, H. T. B.; Jun, D. W.; Mahfoud, Z.; Lin, W.; Bosman, M. Electron dynamics in plasmons. Nanoscale 2021, 13, 2801-2810.

16. Benhayoun, O.; Terekhin, P. N.; Ivanov, D. S.; Rethfeld, B.; Garcia, M. E. Theory for heating of metals assisted by Surface Plasmon Polaritons. Applied Surface Science 2021, 150427.

17. Khurgin, J. B.; Petrov, A.; Eich, M.; Uskov, A. V. Direct Plasmonic Excitation of the Hybridized Surface States in Metal Nanoparticles. ACS Photonics 2021,

18. In this frequency range, the real part of the dielectric function passes zero (epsilon near zero).

19. Hopfield, J. J. Effect of Electron-Electron Interactions on Photoemission in Simple Metals. Physical Review 1965, 139, A419A424.

20. Barman, S. R.; Biswas, C.; Horn, K. Collective excitations on silver surfaces studied by photoyield. Surface Science 2004, 566568, 538-543.

21. Barman, S.; Biswas, C.; Horn, K. Electronic excitations on silver surfaces. Physical Review B 2004, 69, 045413.

22. Reutzel, M.; Li, A.; Gumhalter, B.; Petek, H. Nonlinear Plasmonic Photoelectron
Response of $\mathrm{Ag}(111)$. Physical Review Letters 2019, 123, 017404.

23. Li, A.; Reutzel, M.; Wang, Z.; Novko, D.; Gumhalter, B.; Petek, H. Plasmonic Photoemission from Single-Crystalline Silver. ACS Photonics 2021, 8, 247-258.

24. Novko, D.; Despoja, V.; Reutzel, M.; Li, A.; Petek, H.; Gumhalter, B. Plasmonically assisted channels of photoemission from metals. Physical Review B 2021, 103, 205401.

25. Kale, M. J.; Christopher, P. Plasmons at the interface. Science 2015, 349, 587-588.

26. Tan, S.; Argondizzo, A.; Ren, J.; Liu, L.; Zhao, J.; Petek, H. Plasmonic coupling at a metal/semiconductor interface. Nature Photonics 2017, 11, 806-812.

27. Tan, S.; Dai, Y.; Zhang, S.; Liu, L.; Zhao, J.; Petek, H. Coherent Electron Transfer at the Ag/Graphite Heterojunction Interface. Physical Review Letters 2018, 120, 126801.

28. Shibuta, M.; Yamamoto, K.; Ohta, T.; Inoue, T.; Mizoguchi, K.; Nakaya, M.; Eguchi, T.; Nakajima, A. Confined Hot Electron Relaxation at the Molecular Heterointerface of the Size-Selected Plasmonic Noble Metal Nanocluster and Layered C60. ACS Nano 2021, 15, 1199-1209.

29. Link, S.; El-Sayed, M. A. Spectral Properties and Relaxation Dynamics of Surface Plasmon Electronic Oscillations in Gold and Silver Nanodots and Nanorods. The Journal of Physical Chemistry B 1999, 103, 8410-8426.

30. Foerster, B.; Joplin, A.; Kaefer, K.; Celiksoy, S.; Link, S.; Sönnichsen, C. Chemical Interface Damping Depends on Electrons Reaching the Surface. ACS Nano 2017, 11, 2886-2893.

31. Harutyunyan, H.; Martinson, A. B. F.; Rosenmann, D.; Khorashad, L. K.; 
Besteiro, L. V.; Govorov, A. O.; Wiederrecht, G. P. Anomalous ultrafast dynamics of hot plasmonic electrons in nanostructures with hot spots. Nature Nanotechnology 2015, 10, 770-774.

32. Méjard, R.; Verdy, A.; Petit, M.; Bouhelier, A.; Cluzel, B.; Demichel, O. EnergyResolved Hot-Carrier Relaxation Dynamics in Monocrystalline Plasmonic Nanoantennas. ACS Photonics 2016, 3, 1482-1488.

33. Heilpern, T.; Manjare, M.; Govorov, A. O.; Wiederrecht, G. P.; Gray, S. K.; Harutyunyan, H. Determination of hot carrier energy distributions from inversion of ultrafast pump-probe reflectivity measurements. Nature Communications 2018, 9.

34. Fujimoto, J. G.; Liu, J. M.; Ippen, E. P.; Bloembergen, N. Femtosecond Laser Interaction with Metallic Tungsten and Nonequilibrium Electron and Lattice Temperatures. Physical Review Letters 1984, 53, 1837-1840.

35. Schoenlein, R. W.; Fujimoto, J. G.; Eesley, G. L.; Capehart, T. W. Femtosecond Studies of Image-Potential Dynamics in Metals. Physical Review Letters 1988, 61, 2596-2599.

36. Schmuttenmaer, C. A.; Aeschlimann, M.; Elsayed-Ali, H. E.; Miller, R. J. D.; Mantell, D. A.; Cao, J.; Gao, Y. Time-resolved two-photon photoemission from $\mathrm{Cu}(100)$ : Energy dependence of electron relaxation. Physical Review B 1994, 50, 8957-8960.

37. Petek, H.; Ogawa, S. Femtosecond timeresolved two-photon photoemission studies of electron dynamics in metals. Progress in Surface Science 1997, 56, 239 - 310.

38. Bauer, M.; Marienfeld, A.; Aeschlimann, M. Hot electron lifetimes in metals probed by time-resolved two-photon photoemission. Progress in Surface Science 2015, 90, 319 - 376.

39. Aeschlimann, M.; Bauer, M.; Pawlik, S. Competing nonradiative channels for hot electron induced surface photochemistry. Chemical Physics 1996, 205, 127 - 141.

40. Schmidt, O.; Bauer, M.; Wiemann, C.; Porath, R.; Scharte, M.; Andreyev, O.; Schönhense, G.; Aeschlimann, M. Timeresolved two photon photoemission electron microscopy. Appl Phys B 2002, 74, 223227.

41. Cinchetti, M.; Gloskovskii, A.; Nepjiko, S. A.; Schönhense, G.; Rochholz, H.; Kreiter, M. Photoemission Electron Microscopy as a Tool for the Investigation of Optical Near Fields. Physical Review Letters 2005, 95, 047601.

42. Bayer, D.; Wiemann, C.; Gaier, O.; Bauer, M.; Aeschlimann, M. Time-resolved 2PPE and time-resolved PEEM as a probe of LSP's in silver nanoparticles. Journal of Nanomaterials 2008, 2008.

43. Lemke, C.; Leißner, T.; Evlyukhin, A.; Radke, J. W.; Klick, A.; Fiutowski, J.; Kjelstrup-Hansen, J.; Rubahn, H.-G.; Chichkov, B. N.; Reinhardt, C.; Bauer, M. The Interplay between Localized and Propagating Plasmonic Excitations Tracked in Space and Time. Nano Letters 2014, 14, 2431-2435.

44. Kahl, P.; Wall, S.; Witt, C.; Schneider, C.; Bayer, D.; Fischer, A.; Melchior, P.; Horn-von Hoegen, M.; Aeschlimann, M.; Meyer zu Heringdorf, F.-J. Normal-Incidence Photoemission Electron Microscopy (NI-PEEM) for Imaging Surface Plasmon Polaritons. Plasmonics 2014, 9, 1401.

45. Kahl, P.; Podbiel, D.; Schneider, C.; Makris, A.; Sindermann, S.; Witt, C.; Kilbane, D.; Horn-von Hoegen, M.; Aeschlimann, M.; Meyer zu Heringdorf, F. Direct Observation of Surface Plasmon Polariton Propagation and Interference by TimeResolved Imaging in Normal-Incidence Two Photon Photoemission Microscopy. Plasmonics 2017, 13, 239-246. 
46. Scharte, M.; Porath, R.; Ohms, T.; Aeschlimann, M.; Krenn, J.; Ditlbacher, H.; Aussenegg, F.; Liebsch, A. Do Mie plasmons have a longer lifetime on resonance than off resonance? Applied Physics B 2001, 73, 305-310.

47. Merschdorf, M.; Kennerknecht, C.; Pfeiffer, W. Collective and single-particle dynamics in time-resolved two-photon photoemission. Physical Review B 2004, 70, 193401.

48. Podbiel, D.; Kahl, P.; Makris, A.; Frank, B.; Sindermann, S.; Davis, T. J.; Giessen, H.; von Hoegen, M. H.; zu Heringdorf, F.-J. M. Imaging the Nonlinear Plasmoemission Dynamics of Electrons from Strong Plasmonic Fields. Nano Letters 2017, 17, 6569-6574.

49. Lehr, M.; Foerster, B.; Schmitt, M.; Krüger, K.; Sönnichsen, C.; Schönhense, G.; Elmers, H.-J. Momentum Distribution of Electrons Emitted from Resonantly Excited Individual Gold Nanorods. Nano Letters 2017, 17, 6606-6612.

50. Lehr, M.; Bley, K.; Vogel, N.; Rethfeld, B.; Schönhense, G.; Elmers, H.-J. Evidence of Spatially Inhomogeneous Electron Temperature in a Resonantly Excited Array of Bow-Tie Nanoantennas. The Journal of Physical Chemistry C 2019, 123, 1242912436.

51. Pettine, J.; Meyer, S. M.; Medeghini, F.; Murphy, C. J.; Nesbitt, D. J. Controlling the Spatial and Momentum Distributions of Plasmonic Carriers: Volume vs Surface Effects. ACS Nano 2021, 15, 1566-1578.

52. Spektor, G.; Kilbane, D.; Mahro, A.; Hartelt, M.; Prinz, E.; Aeschlimann, M.; Orenstein, M. Mixing the Light Spin with Plasmon Orbit by Nonlinear Light-Matter Interaction in Gold. Physical Review $X$ 2019, 9, 021031.
53. A corresponding pulse propagating in negative $x$-direction is also launched from the slit, but it is outside of the detection area.

54. The exact scaling depends on dephasing of the coherences along the excitation pathway.

55. Terekhin, P. N.; Benhayoun, O.; Weber, S. T.; Ivanov, D. S.; Garcia, M. E.; Rethfeld, B. Influence of surface plasmon polaritons on laser energy absorption and structuring of surfaces. Applied Surface Science 2020, 512, 144420.

56. Joly, A. G.; El-Khoury, P. Z.; Hess, W. P. Spatiotemporal Imaging of Surface Plasmons Using Two-Color Photoemission Electron Microscopy. The Journal of Physical Chemistry C 2018, 122, 20981-20988.

57. Kotsugi, M.; Kuch, W.; Offi, F.; Chelaru, L. I.; Kirschner, J. Microspectroscopic two-dimensional Fermi surface mapping using a photoelectron emission microscope. Review of Scientific Instruments 2003, 74, 2754-2758.

58. Tusche, C.; Goslawski, P.; Kutnyakhov, D.; Ellguth, M.; Medjanik, K.; Elmers, H. J.; Chernov, S.; Wallauer, R.; Engel, D.; Jankowiak, A.; Schönhense, G. Multi$\mathrm{MHz}$ time-of-flight electronic bandstructure imaging of graphene on $\operatorname{Ir}(111) . A p$ plied Physics Letters 2016, 108, 261602.

59. Haag, F.; Eul, T.; Thielen, P.; Haag, N.; Stadtmüller, B.; Aeschlimann, M. Timeresolved two-photon momentum microscopy - A new approach to study hot carrier lifetimes in momentum space. Review of Scientific Instruments 2019, 90, 103104.

60. Maklar, J.; Dong, S.; Beaulieu, S.; Pincelli, T.; Dendzik, M.; Windsor, Y. W.; Xian, R. P.; Wolf, M.; Ernstorfer, R.; Rettig, L. A quantitative comparison of timeof-flight momentum microscopes and hemispherical analyzers for time- and angleresolved photoemission spectroscopy exper- 
iments. Review of Scientific Instruments 2020, 91, 123112.

61. Wu, K.; Chen, J.; McBride, J. R.; Lian, T. Efficient hot-electron transfer by a plasmon-induced interfacial charge-transfer transition. Science 2015, 349, 632-635.

62. Therrien, A. J.; Kale, M. J.; Yuan, L.; Zhang, C.; Halas, N. J.; Christopher, P. Impact of chemical interface damping on surface plasmon dephasing. Faraday Discussions 2019, 214, 59-72.

63. Foerster, B.; Hartelt, M.; Collins, S. S. E.; Aeschlimann, M.; Link, S.; Sönnichsen, C. Interfacial States Cause Equal Decay of Plasmons and Hot Electrons at Gold-Metal Oxide Interfaces. Nano Letters 2020, 20, 3338-3343.

64. Olmon, R. L.; Slovick, B.; Johnson, T. W.; Shelton, D.; Oh, S.-H.; Boreman, G. D.; Raschke, M. B. Optical dielectric function of gold. Physical Review B 2012, 86, 235147.

65. Oelsner, A.; Rohmer, M.; Schneider, C.; Bayer, D.; Schönhense, G.; Aeschlimann, M. Time- and energy resolved photoemission electron microscopy-imaging of photoelectron time-of-flight analysis by means of pulsed excitations. Journal of Electron Spectroscopy and Related Phenomena 2010, 178 - 179, 317 - 330. 


\title{
Energy and Momentum Distribution of Surface Plasmon-induced Hot Carriers Isolated via Spatiotemporal Separation
}

\section{Supplementary Information}

\begin{abstract}
Michael Hartelt, ${ }^{*}$ Pavel N. Terekhin, Tobias Eul, Anna-Katharina Mahro, Benjamin
Frisch, Eva Prinz, Baerbel Rethfeld, Benjamin Stadtmüller, and Martin Aeschlimann Department of Physics and Research Center OPTIMAS, TU Kaiserslautern, Germany
\end{abstract}

(Dated: August 2, 2021)

\section{REAL SPACE IMAGES OF THE SAMPLE}
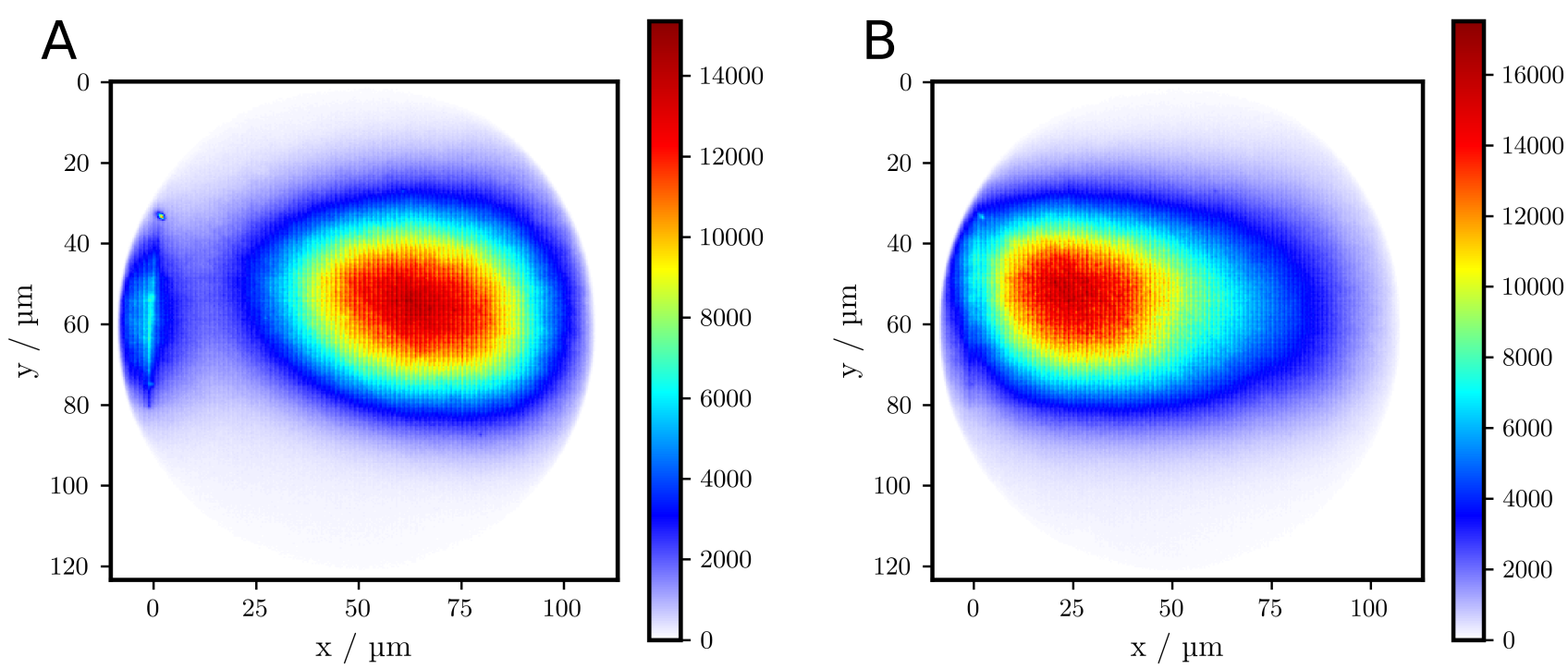

Figure S1. Real space PEEM images of the sample. A) PEEM image of the static probe contribution $Y_{\text {probe }}$ to the photoemission signal, taken from the full $4 \mathrm{D}$ dataset for $\Delta t=-300 \mathrm{fs}$ to $-200 \mathrm{fs}$ (no temporal overlap), integrated over all electron energies. Mainly visible is the spatial profile of the probe pulse on the right side ("Blue 2PPE") and the excitation slit at $x=0 \mu \mathrm{m}$ illuminated by the pump pulse on the left side ("Red 3PPE"). B) PEEM image, taken from the full 4D dataset for $\Delta t=-10 \mathrm{fs}$ to $10 \mathrm{fs}$ (time zero), integrated over all electron energies. The enhanced yield between the coupling edged and the probe pulse comes from electrons excited by the pump pulse and photo-emitted by the probe pulse ("Red-Blue 2PPE"). 


\section{COMPONENTS OF THE THEORETICAL SOURCE TERM}
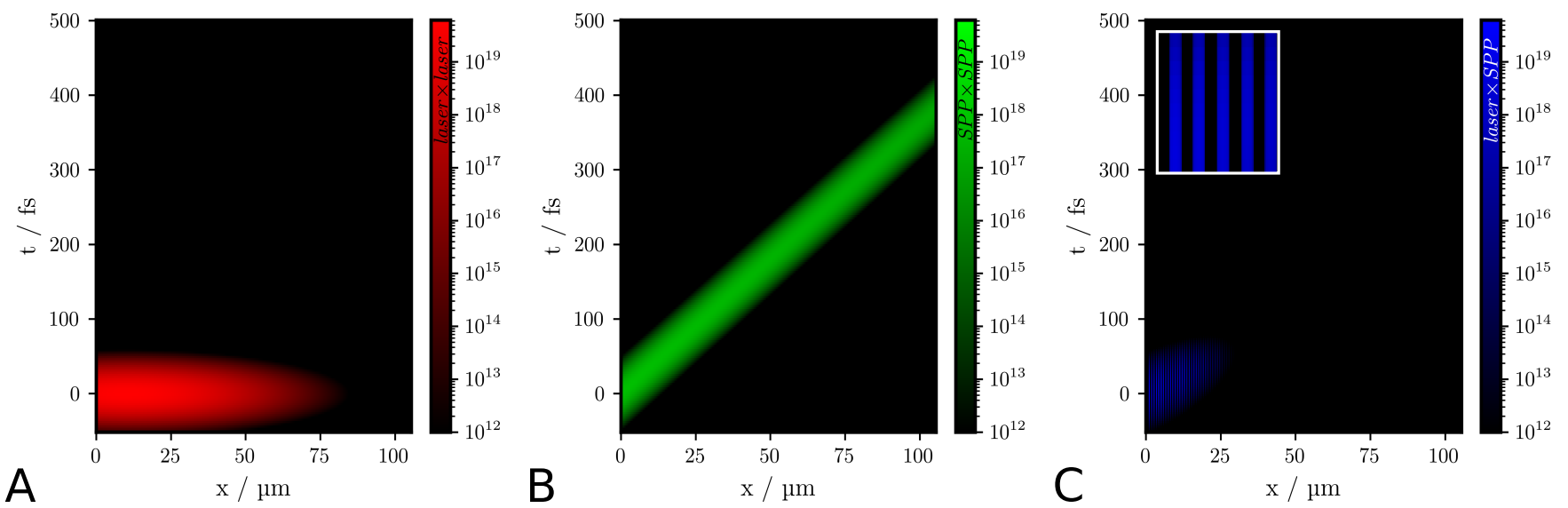

Figure S2. Calculation of the source term components A) laser-laser, B) SPP-SPP, and C) laser-SPP. The time-dependent energy density of the electromagnetic field contributions at the surface is shown for an excitation pulse with $\lambda=800 \mathrm{~nm}$ and a pulse duration of $23 \mathrm{fs}$. The pulse maximum arrives at the sample surface at $t=0 \mathrm{fs}$. The colorbars are given in $\mathrm{J} \mathrm{s}^{-1} \mathrm{~m}^{-3}$. The inset in C) is a zoom of $x=10 \mu \mathrm{m}$ to $15 \mu \mathrm{m}$ and $t=15 \mathrm{fs}$ to $45 \mathrm{fs}$, showing the striped interference pattern.

\section{CALCULATION OF THE OBSERVED SPP VELOCITY}

The real part of the SPP wave vector $k_{\mathrm{SPP}}^{\prime}$ from the solution of the wave equation at the interface ${ }^{\mathrm{S} 1}$ is given by

$$
k_{\mathrm{SPP}}^{\prime}=\Re\left(\frac{\omega}{c_{0}} \cdot \sqrt{\frac{\epsilon_{\mathrm{m}} \epsilon_{\mathrm{d}}}{\epsilon_{\mathrm{m}}+\epsilon_{\mathrm{d}}}}\right),
$$

while the probe pulse counter-propagates with an in-plane wave vector of

$$
k_{\|}=-k_{\text {probe }} \sin (\mathrm{AOI})=-\frac{\omega}{c_{0}} \sin (\mathrm{AOI}),
$$

the negative sign signifies the orientation of the projected wave vector in negative $x$-direction.

This results in the well-known effect for SPP waves observed in PEEM, ${ }^{\mathrm{S} 2}$ the so-called beating pattern

$$
k_{\text {perceived }}=k_{\mathrm{SPP}}^{\prime}-k_{\|} .
$$

We can link the perceived SPP group velocity $v_{\text {perceived }}$ and the actual SPP group velocity $v_{\text {SPP }}$ by taking the derivative over the frequency $\omega$ from equation S3:

$$
\frac{1}{v_{\text {perceived }}}=\frac{d k_{\text {perceived }}}{d \omega}=\frac{d k_{\mathrm{SPP}}^{\prime}}{d \omega}-\frac{d k_{\|}}{d \omega}=\frac{1}{v_{\mathrm{SPP}}}+\frac{\sin (\mathrm{AOI})}{c_{0}} .
$$

Solving for $v_{\text {perceived }}$ yields the relation used in the main manuscript:

$$
v_{\text {perceived }}=v_{\mathrm{SPP}} \cdot \frac{1}{1+\frac{v_{\mathrm{SPP}}}{c_{0}} \sin (\mathrm{AOI})} .
$$

\section{RAW SPECTRA AND DIFFERENCE SPECTRUM}

The photon-induced and plasmon-induced hot electron spectra from the $\Delta t$-Energy-plot in the main manuscript are normalized to their work function edges at the low-energy cutoff for $E-E_{\mathrm{F}}=0.6 \mathrm{eV}$. For reference, the raw 


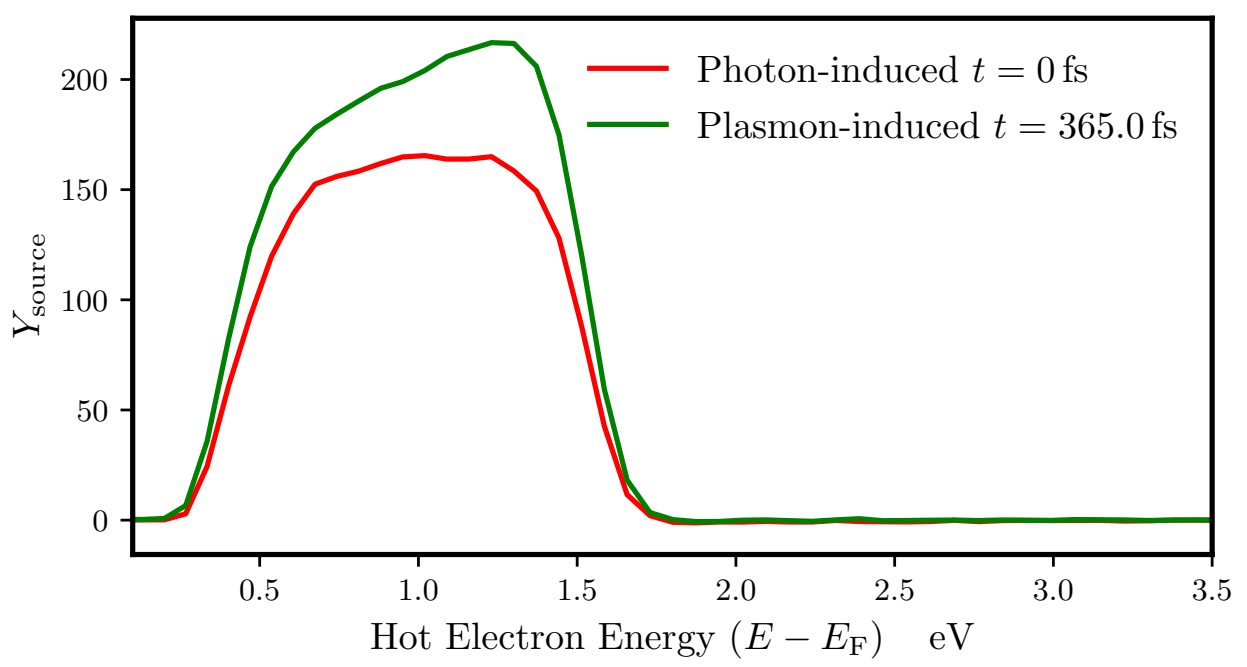

Figure S3. Raw Spectra from the $\Delta t$-Energy-plot in the main manuscript, without normalization. The $Y_{\text {source }}$ values are given in counts.

spectra without normalization are given in Figure S3. The difference in absolute height is caused by the strength of the respective source field.

The two normalized curves from the main manuscript were subtracted for Figure S4. The main feature is the plasmonic excitation peak at high energies. According to the reasoning in the main manuscript, this would correspond to the electron spectrum of the plasmon aspect of SPP. The smaller peak in the low-energy cutoff region, representing a slight difference in the shape of the work function edge, is most likely caused by the SPP propagation through the finite real space integration area. As discussed in Section VI this causes an artifact of increased detection of secondary electrons.

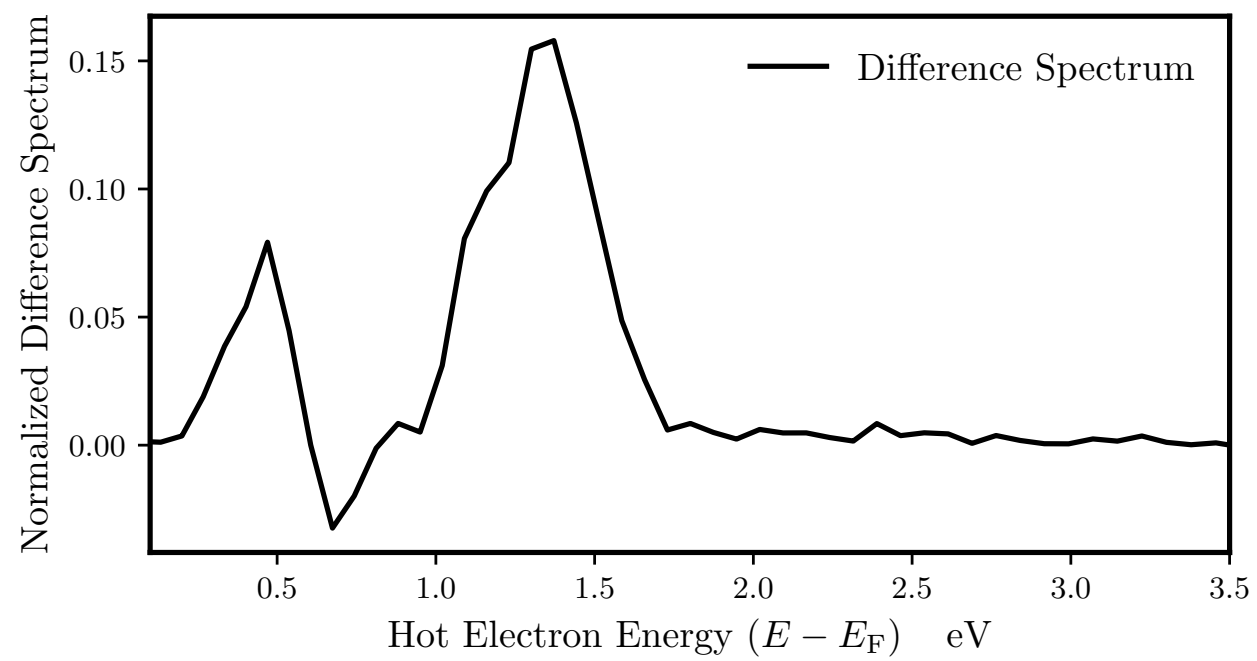

Figure S4. Difference Spectrum between $Y_{\text {source }}\left(\Delta t_{\mathrm{SPP}}\right)$ and $Y_{\text {source }}\left(\Delta t_{0}\right)$ as normalized in the main manuscript. 


\section{MOMENTUM MICROSCOPY DATA}

The momentum microscopy plots in figures S5, S6, and S7 were shifted, noise-filtered and binned as described in under Data Evaluation in the Methods section in the main manuscript.

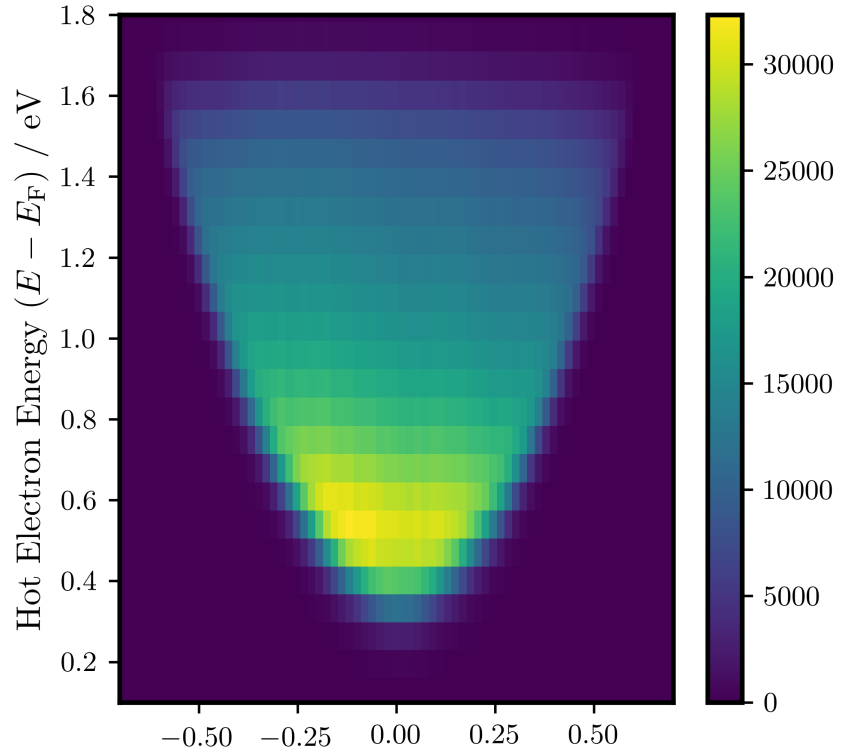

A: photon $k_{x} / \AA^{-1}$

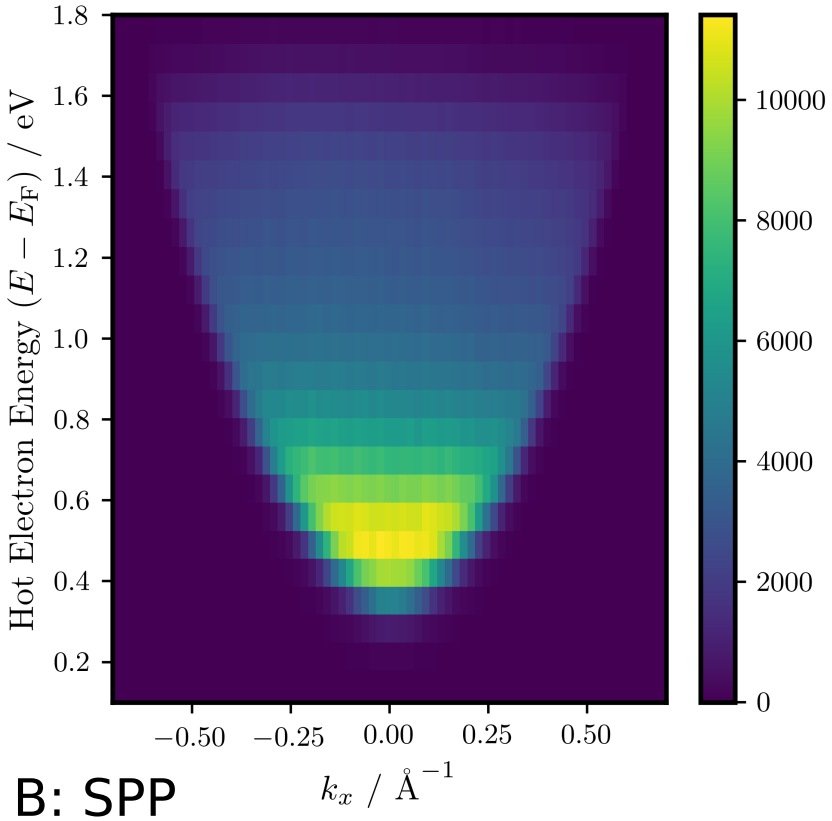

Figure S5. Momentum microscopy data A) $Y_{\text {photon }}\left(k_{x}, k_{y}, E\right)$ and B) $Y_{\mathrm{SPP}}\left(k_{x}, k_{y}, E\right)$ along the $E$ - $k_{x}$-direction for $k_{y}=0 \AA^{-1}$.

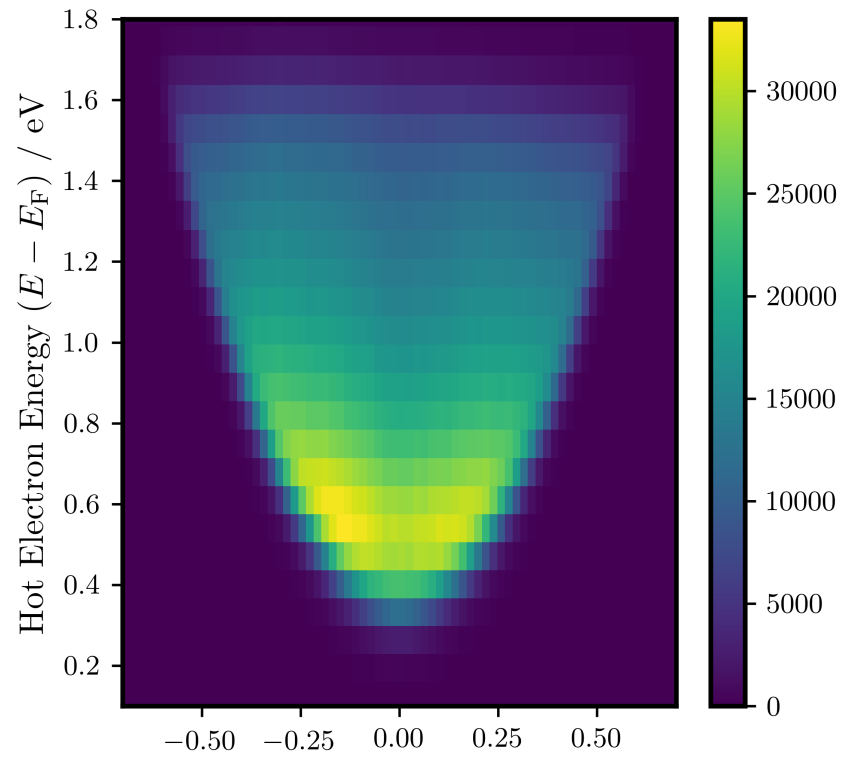

A: photon $k_{y} / \AA^{-1}$

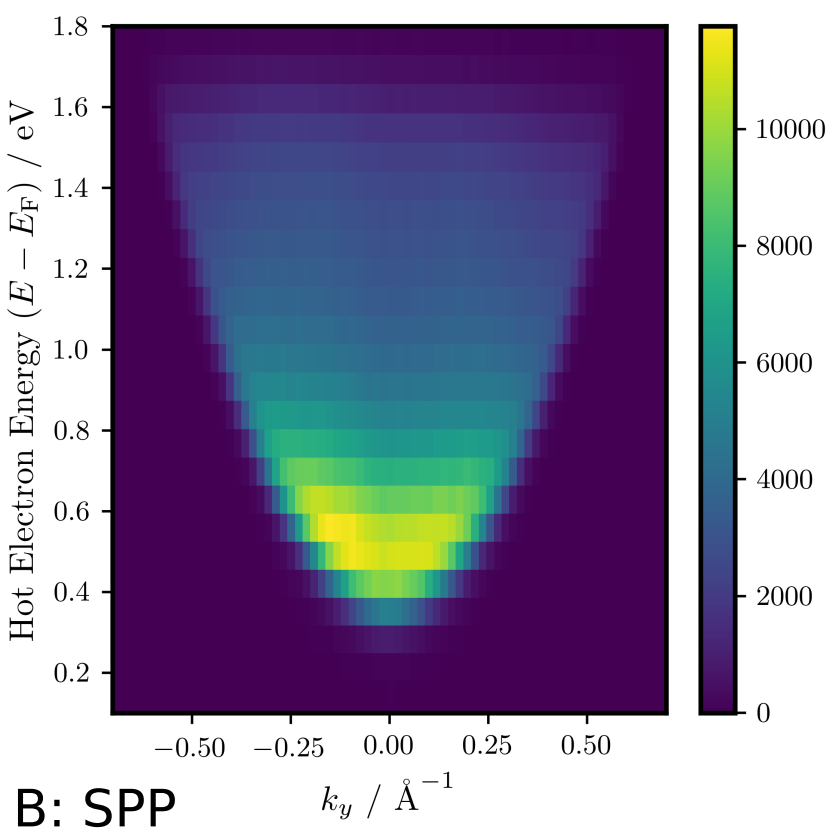

Figure S6. Momentum microscopy data A) $Y_{\text {photon }}\left(k_{x}, k_{y}, E\right)$ and B) $Y_{\mathrm{SPP}}\left(k_{x}, k_{y}, E\right)$ along the $E$ - $k_{y}$-direction for $k_{x}=0 \AA^{-1}$. 


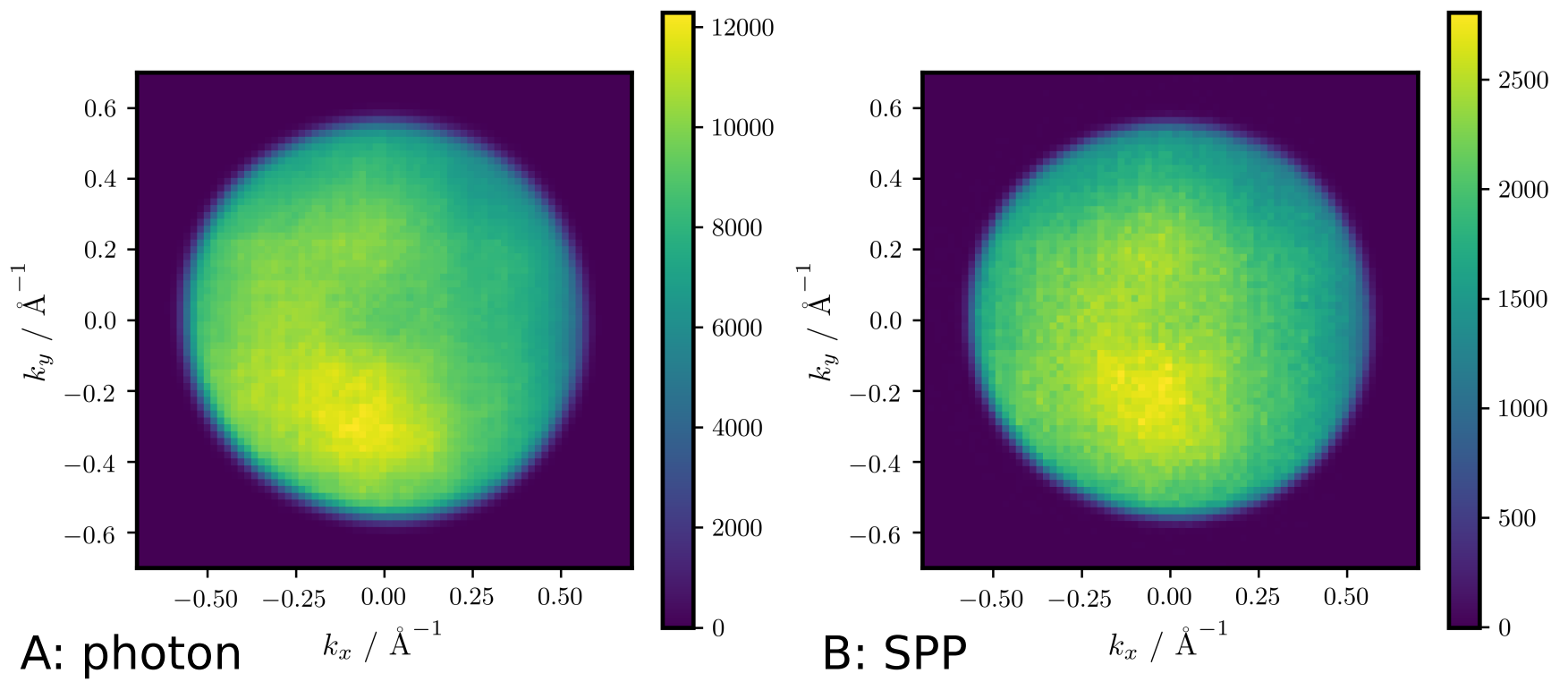

Figure S7. Momentum microscopy data A) $Y_{\text {photon }}\left(k_{x}, k_{y}, E\right)$ and B) $Y_{\mathrm{SPP}}\left(k_{x}, k_{y}, E\right)$ along the $k_{x}-k_{y}$-direction for $E-$ $E_{\mathrm{F}}=1.4 \mathrm{eV}$ to $1.5 \mathrm{eV}$.

\section{COMPENSATION OF THE SPP PROPAGATION ARTIFACT IN THE MOMENTUM DISTRIBUTION}
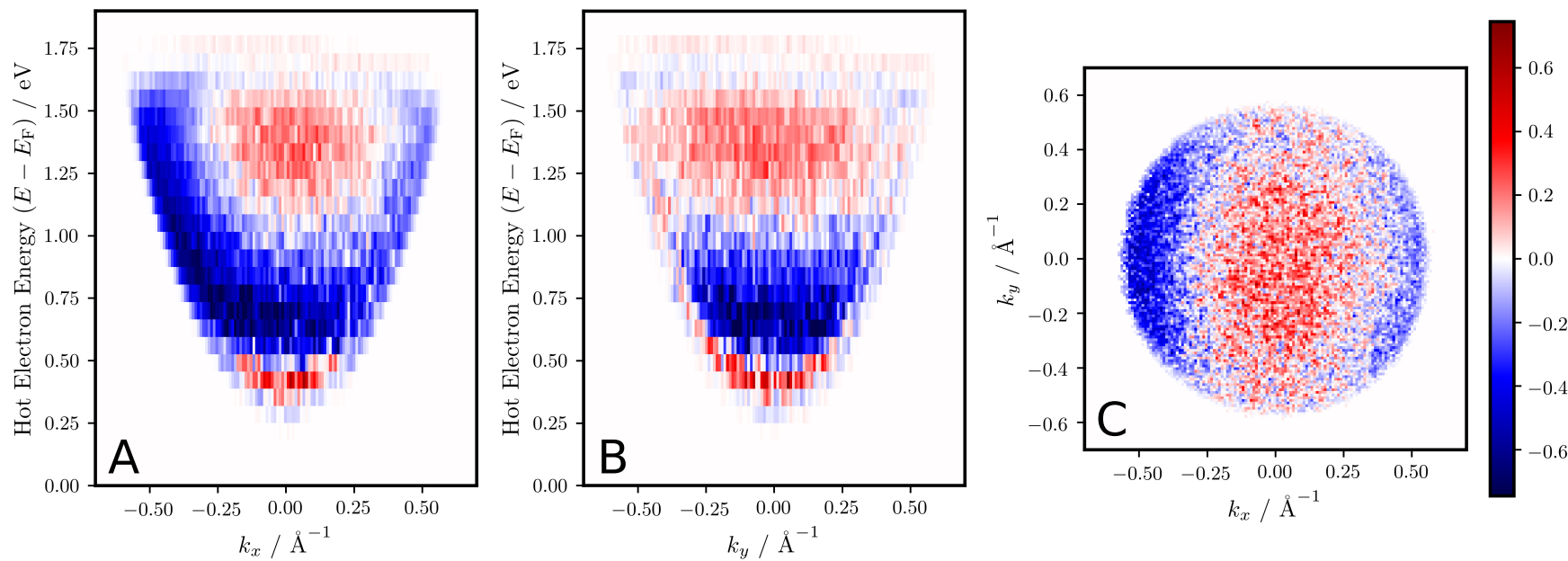

Figure S8. Momentum distribution of plasmon-induced hot electrons relative to photon-induced hot electrons, mitigating the apparent broadening effect by the propagation of the SPP pulse through the field-of-view. Plotted are cuts of the normalized difference values $\Delta Y\left(k_{x}, k_{y}, E\right)$ along A) the $E$ - $k_{x}$-direction for $\left.k_{y}=0 \AA^{-1}, \mathrm{~B}\right)$ the $E$ - $k_{y}$-direction for $k_{x}=0 \AA^{-1}$, and C) the $k_{x}-k_{y}$-direction for $E-E_{\mathrm{F}}=1.4 \mathrm{eV}$ to $1.5 \mathrm{eV}$.

As described in the main manuscript, the apparent low-energy enhancement of plasmon-induced electrons in the $k$-space data is caused by an apparent broadening of the SPP pulse duration due to its propagation through the detection area. This is an observation artifact caused by the experimental scheme and setup, and not to be confused with a real, dispersive pulse broadening. (Due to the very linear SPP dispersion over the used wavelength range, the dispersive broadening during the propagation time is small.) To mitigate this effect, a measurement with reduced aperture size of $\approx 18 \mu \mathrm{m}$ was performed, recording the full time trace of $\Delta t=-334 \mathrm{fs}$ to $800 \mathrm{fs}$. The evaluation was carried out as described in the main manuscript, but using integration ranges instead of fixed values for the respective delay times: $\Delta t_{\mathrm{SPP}}=345 \mathrm{fs}$ to $355 \mathrm{fs}, \Delta t_{\text {photon }}=-80 \mathrm{fs}$ to $80 \mathrm{fs}$, and $\Delta t_{\text {probe }}=-334 \mathrm{fs}$ to $-167 \mathrm{fs}$. In this way, a 
prolonged time window for the photonic signal compensates for the propagation time of the SPP pulse through the detection area. To select only the region of statistically relevant data, voxels with less than 80 counts in $Y_{\mathrm{SPP}}$ or less than 5 counts in $Y_{\text {photon }}$ were ignored.

The result in Figure S8 shows that the larger integration range for the photonic signal compensates for the propagation artifact and the signal enhancement caused by secondary electrons is mitigated. The high-energy feature remains, but it is deformed along the $k_{y}$-direction. This deformation is caused by an electron-optical artifact when the iris aperture is pulled to very small diameters. It is then no longer symmetric, but a hexagonal shape elongated along the $y$-direction.

\section{MOVIES}

- Animation of the Scheme of Spatiotemporal Separation spatiotemporal_scheme_animated.mkv

- Movie of the Spatiotemporal Dynamics Plasmon-induced Hot Carriers. Shown is $Y_{\text {source }}(x, y, E, \Delta t)$ for an electron energy of $E=1.5 \mathrm{eV}$. The field-of-view and axes are as depicted in Figure S1.

timeseries_binned_fermi.avi

* hartelt@physik.uni-kl.de

[S1] S. A. Maier, Plasmonics: fundamentals and applications: fundamentals and applications (Springer Science \& Business Media, 2007).

[S2] A. Kubo, N. Pontius, and H. Petek, Nano Letters 7, 470 (2007). 